\title{
Mixtures of multiplicative cascade models in geochemistry
}

\author{
F. P. Agterberg \\ Geological Survey of Canada, 601 Booth Street, Ottawa, K1A0E8, Canada \\ Received: 5 February 2007 - Revised: 11 May 2007 - Accepted: 13 May 2007 - Published: 23 May 2007
}

\begin{abstract}
Multifractal modeling of geochemical map data can help to explain the nature of frequency distributions of element concentration values for small rock samples and their spatial covariance structure. Useful frequency distribution models are the lognormal and Pareto distributions which plot as straight lines on logarithmic probability and $\log -\log$ paper, respectively. The model of de Wijs is a simple multiplicative cascade resulting in discrete logbinomial distribution that closely approximates the lognormal. In this model, smaller blocks resulting from dividing larger blocks into parts have concentration values with constant ratios that are scale-independent. The approach can be modified by adopting random variables for these ratios. Other modifications include a single cascade model with ratio parameters that depend on magnitude of concentration value. The Turcotte model, which is another variant of the model of de Wijs, results in a Pareto distribution. Often a single straight line on logarithmic probability or log-log paper does not provide a good fit to observed data and two or more distributions should be fitted. For example, geochemical background and anomalies (extremely high values) have separate frequency distributions for concentration values and for local singularity coefficients. Mixtures of distributions can be simulated by adding the results of separate cascade models. Regardless of properties of background, an unbiased estimate can be obtained of the parameter of the Pareto distribution characterizing anomalies in the upper tail of the element concentration frequency distribution or lower tail of the local singularity distribution. Computer simulation experiments and practical examples are used to illustrate the approach.
\end{abstract}

Correspondence to: F. P. Agterberg

(agterber@nrcan.gc.ca)

\section{Introduction}

1.1 Lognormal and pareto frequency distribution models

Two frequency distribution models frequently used in geochemistry and natural resource analysis are the lognormal and Pareto. Both models can provide good approximations for chemical element concentration values of rock samples, the sizes and grades of ore deposits, or the sizes of oil and gas pools. The lognormal has a longer history of application in these fields than the Pareto, leading Mandelbrot (1983) to pose a challenge to the geoscience community by stating that oil and other resources have Pareto distributions, and that this "finding disagrees with the dominant opinion, that the quantities in question are lognormally distributed. The difference is extremely significant, the reserves being much higher under the hyperbolic than under the lognormal law."

The preceding statement is graphically illustrated in Fig. 1. In Fig. 1a a hypothetical lognormal distribution for amount of metal per ore deposit plots as a curve on log-log paper. The straightline that is approximately tangent to the lognormal would represent a Pareto distribution. In Fig. 1b the same lognormal plots as a straightline on logarithmic probability paper whereas the Pareto of Fig. 1a has become a curve. The curvatures in these two diagrams are such that the Pareto has a high-value tail that is thicker than that of the lognormal. For low values, Pareto frequencies also exceed those of the lognormal.

In a later paper concerned with multifractal measures for the geoscientist, and assuming that ore tonnage is equivalent to volume, Mandelbrot (1989) uses the model of de Wijs (1951) as a starting point for spatial distribution of metals in the Earth and concludes that ore "curdles" into a binomial multifractal. The model of de Wijs also will be taken as a starting point in this paper. However, it generally leads to lognormal distribution of metal concentrations without

Published by Copernicus Publications on behalf of the European Geosciences Union and the American Geophysical Union. 


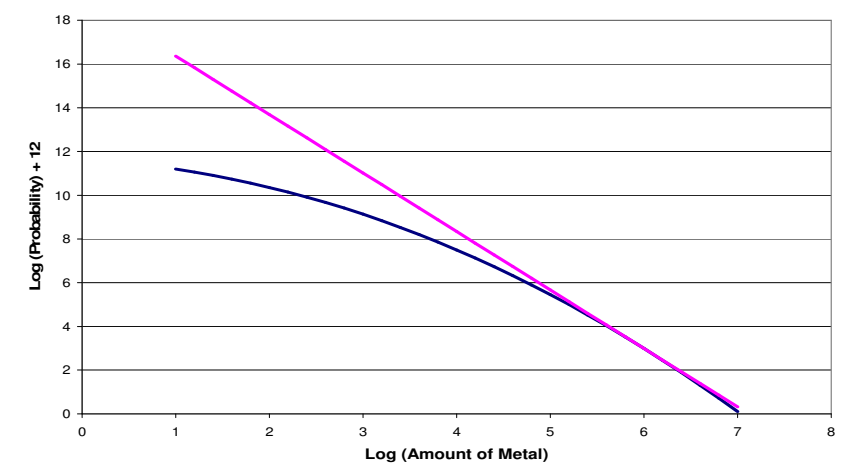

Fig. 1a. Lognormal on log-log paper; artificial example (Log base 10).

Pareto tails and further modifications of the approach are needed.

The model of de Wijs is a simple example of a binomial multiplicative cascade (cf. Mandelbrot, 1989). The theory of cascades was developed extensively over the past 20 years by Lovejoy and Schertzer (1991) and Schertzer et al. (1997), and other geophysicists, particularly in connection with cloud formation and rainfall (Schertzer and Lovejoy, 1987; Over and Gupta, 1996; Veneziano and Furcolo, 2003; Veneziano and Langousis, 2005). More recently, multifractal modeling of solid-Earth processes has been advanced by Cheng and colleagues (Cheng, 1994; Cheng and Agterberg, 1996; Cheng, 1995; Cheng, 2005).

In applications concerned with turbulence, the original binomial model of de Wijs is known as the $p$-model (Schertzer et al., 1997). Several advanced cascade models in meteorology (Veneziano and Langousis, 2005) result in frequency distributions that resemble the lognormal but have Pareto tails. The Pareto is characterized by a single parameter that can be related to a fractal dimension. The lognormal has two parameters. A basic difference between the two models is that Pareto frequency density approaches infinity in its lowvalue tail whereas lognormal frequency density at zero-value is zero. During the last 20 years it became increasingly clear that the Pareto generally performs better than the lognormal in modeling the upper-value tails of frequency distributions in geochemistry and resource analysis. Turcotte $(1997,2002)$ has developed a variant of the model of de Wijs that results in a Pareto distribution, which is truncated in its lower tail.

In practical applications, frequency density at zero-value is observed to be zero. Thus the lognormal generally provides a more realistic model for modeling low-value tails. This is probably one reason why the lognormal was preferred to the Pareto in the past. The other reason was that, in practical applications, the largest values in upper tails of frequency distributions become increasingly rare when value goes to infinity. In goodness-of-fit tests the largest values generally are combined into a single class so that it is not possible to distinguish between lognormal and Pareto (Agterberg, 1995).

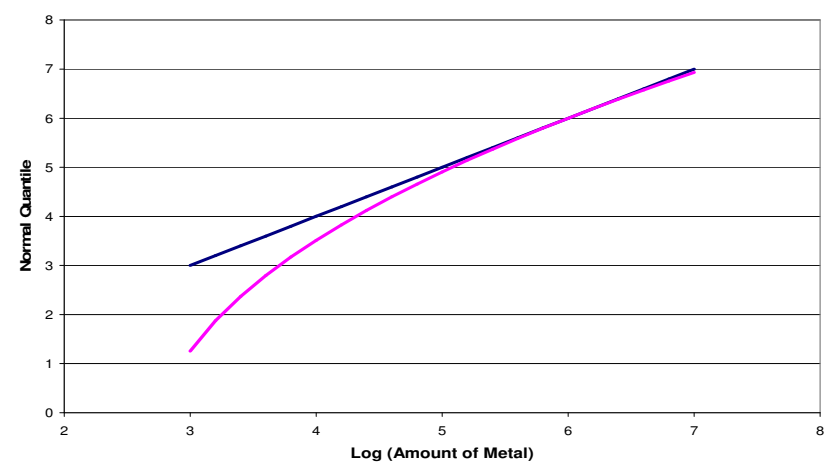

Fig. 1b. Pareto of Fig. 1a on lognormal Q-Q plot.

This paper is concerned with combining data from large regions. The problem of interest is how to explain and model a regional frequency distribution of element concentration values that resembles the lognormal but displays a powerlaw tail. Two ways to solve this problem are (1) to adopt a single-process model with an end product that satisfies both conditions, and (2) to consider the end product to be a mixture of two or more separate processes resulting in lognormal and Pareto distributions.

Single-process models include the previously mentioned meteorological models (e.g. beta-lognormal cascades of Veneziano and Langousis, 2005). Already in the 1980s, Schertzer and Lovejoy (1985) had pointed out that the $p$ model can be regarded as "micro-canonical" version of their $\alpha$-model in which the strict condition of local preservation of mass is replaced by a more general condition of preservation of mass within wider neighborhoods (preservation of ensemble averages). Cascades of this type can result in pure lognormals and in lognormals with Pareto tails. The applicability of these approaches to geological processes that took place within the Earth's crust remains to be considered. In this paper, a simple variant of the model of de Wijs that results in a lognormal with relatively thick upper tail will be investigated. With respect to mixtures of cascades, the most promising approach consists of superimposing Turcotte's Paretotype models on a lognormal background.

\subsection{Lognormality of Trace elements in rocks}

One of the basic assumptions in geochemical abundance models Brinck, 1974; Harris, 1984; Garrett, 1986) is that trace elements are lognormally distributed. Ahrens (1953) postulated lognormality as the first law of geochemistry. In general, it can not be assumed that the element concentration values for very small blocks of rock collected from a very large environment satisfy a single lognormal frequency distribution model. However, the lognormal model often provides a valid first approximation especially for trace elements. Reasons why the lognormal model may not be applicable include the following: Concentration values for all 
constituents form a closed number system and this prevents major constituents from being lognormally distributed. Also, discrete boundaries (contacts) between different rock types commonly exist in regional applications and mixtures of two or more lognormals would occur if rock types have lognormals with different parameters.

Three types of generating mechanisms or explanations have been suggested for lognormality. The first one is reviewed by Aitchison and Brown (1957): processes during which random increases in value are proportional to value can result in lognormal distributions, in the same way that processes subject to conditions underlying the central limit theorem of mathematical statistics lead to normal distributions. The second type of explanation was first advocated by Vistelius (1960): mixtures of populations with mean values that are proportional to standard deviations can result in positively skewed distributions that resemble lognormal distributions even if the original populations are normal. Finally, multiplicative cascade models such as the model of de Wijs can help to explain lognormality (cf. Agterberg, 2001a and 2007). Allègre and Lewin (1995) provided an overview of geochemical distributions that are either lognormal or Pareto.

A relatively simple generalization of the lognormal model is to assume that the concentration values for a chemical element in a large region or environment originate from two different lognormal populations representing background and anomalies, respectively. The largest concentration values then primarily represent the anomalies. This type of modeling either uses lognormal Q-Q plots (Sinclair, 1991), or use is made of concentration-area (C-A) log-log plots (Cheng et al., 1994) to distinguish between two or more separate populations. Often it can be assumed (Agterberg, 2007) that (a) the relatively small concentration values (background) represent a mixture of different populations, and (b) the largest values satisfy a Pareto distribution with a tail that is thicker than lognormal.

\section{Multiplicative cascade models}

\subsection{Two simple models}

Two relatively simple 2-dimensional multiplicative cascade models are the model of de Wijs (1951) and Turcotte's (1997) "fractal" cascade. These models are graphically illustrated in Figs. 2-4. In the original model of de Wijs, any block of rock is divided into two equal parts. The concentration value $(\xi)$ of a chemical element in the block then can be written as $(1+d) \times \xi$ for one half and $(1-d) \times \xi$ for the other half so that total mass is preserved. The coefficient of dispersion $d$ is independent of block size. The approach can be modified by replacing $d$ by a random variable (random-cut model; Agterberg, 2007). The multifractal patterns of local concentration values generated by this cascade and its generalized version have many local maxima and minima (Fig. 3).

$$
\begin{array}{cc}
(2 \times 2) & \text { squares } \\
(1+d)^{2} & (1+d)(1-d) \\
(1+d)(1-d) & (1-d)^{2}
\end{array}
$$

(4x4) squares

$$
\begin{aligned}
& \begin{array}{llll}
(1+d)^{4} & (1+d)^{3}(1-d) \quad(1+d)^{3}(1-d) \quad(1+d)^{2}(1-d)^{2}
\end{array} \\
& \begin{array}{llll}
(1+d)^{3}(1-d) & (1+d)^{2}(1-d)^{2} & (1+d)^{2}(1-d)^{2} & (1+d)(1-d)^{3}
\end{array} \\
& \begin{array}{llll}
(1+d)^{3}(1-d) & (1+d)^{2}(1-d)^{2} & (1+d)^{2}(1-d)^{2} & (1+d)(1-d)^{3}
\end{array} \\
& \begin{array}{llll}
(1+d)^{2}(1-d)^{2} & (1+d)(1-d)^{3} & (1+d)(1-d)^{3} & (1-d)^{4}
\end{array} \\
& \begin{array}{cccccccc}
\multicolumn{4}{c}{\text { Non-Random }} & \multicolumn{4}{c}{\text { Random }} \\
40 & 31 & 31 & 22 & 04 & 13 & 22 & 31 \\
31 & 22 & 22 & 13 & 13 & 22 & 13 & 22 \\
31 & 22 & 22 & 13 & 13 & 22 & 40 & 31 \\
22 & 13 & 13 & 04 & 22 & 31 & 31 & 22
\end{array}
\end{aligned}
$$

Fig. 2a. First stages of 2-D multiplicative cascade model of de Wijs. Overall average concentration value is equal to 1 ; $d$ is dispersion coefficient. Non-Random index matrix corresponds to $(4 \times 4)$ squares distribution of concentration values.

$$
\begin{aligned}
& \text { (2x2) squares } \\
& (1+d)^{2} \quad(1-d) \\
& (1+d)(1-d) \quad(1-d) \\
& \begin{array}{cccc}
\multicolumn{5}{c}{(\mathbf{4} \mathbf{4}) \text { squares }} \\
(1+d)^{4} & (1+d)^{2}(1-d) & (1-d) & (1-d) \\
(1+d)^{3}(1-d) & (1+d)^{2}(1-d) & (1-d) & (1-d) \\
(1+d)(1-d) & (1+d)(1-d) & (1-d) & (1-d) \\
(1+d)(1-d) & (1+d)(1-d) & (1-d) & (1-d)
\end{array}
\end{aligned}
$$

Fig. 2b. Turcotte's variant of model of de Wijs as in Fig. 2a.

Figure 2a illustrates the model of de Wijs: any cell containing a chemical element in 1-, 2-, or 3-dimensional space with average concentration value $\xi$ set equal to unity is divided into two halves with element concentration values $(1+d) \xi$ and $(1-d) \xi$. For the first cell at the beginning of the process, $\xi$ can be set equal to unity. This implies that all concentration values are divided by their overall regional average concentration value $(m)$. The index of dispersion $(d)$ is independent of cell-size. In 2-D space, two successive subdivisions into quarters result in 4 and 16 cells with concentration values. The maximum element concentration value after $k$ subdivisions is $(1+d)^{k}$, and the minimum value is $(1-d)^{k} ; k$ is even in 2-dimensional applications in order to preserve mass but the frequency distribution of all 


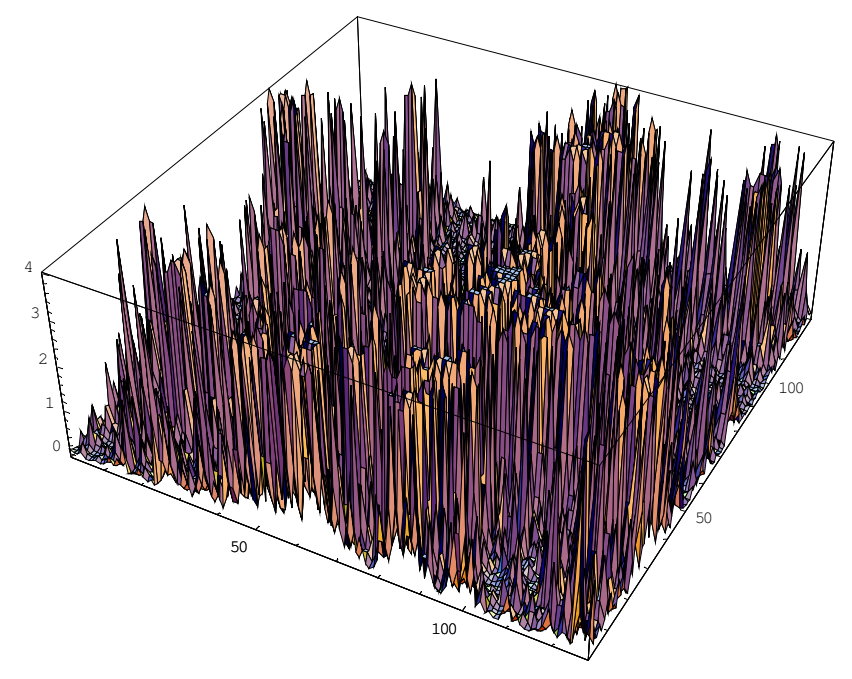

Fig. 3. Realization of model of de Wijs (see Fig. 2, left side) in 2-D for $d=0.4$ and $N=14$. Overall average value is equal to 1 . Values greater than 4 were truncated.

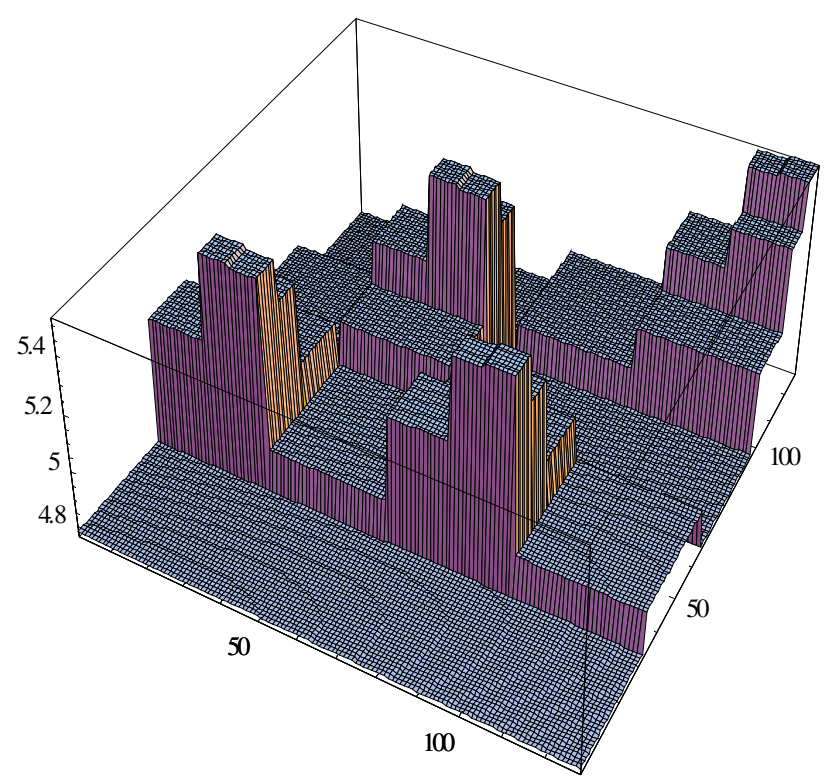

Fig. 4. Four 2-D realizations of Turcotte's fractal model (Fig. 2b) for $d=0.4$ and $N=12$. Total area was subdivided into four quadrants. Vertical scale is for $5+\log _{10}$ (Value).

concentration cannot be distinguished from that arising in 1or 3-dimensional applications of this multiplicative cascade model. In a random cascade, larger and smaller values are assigned to cells using a discrete random variable.

Multifractal patterns generated by a random cascade have more than a single maximum. The frequency distribution of the element concentrations at any stage of this process is called "logbinomial" because logarithmically transformed concentration values satisfy a binomial distribution. The log- binomial converges to a lognormal distribution although its upper and lower value tails remain weaker than those of the lognormal (Agterberg, 2007). Notation can be simplified by using indices that are powers of $(1+d)$ and $(1-d)$, respectively; for example, $(1+d)^{3}(1-d)$ is written as 31 in the 16cell matrix on the left in the next row. If at each stage of subdivision, the location of higher and lower concentration cells is determined by a Bernoulli-type random variable, the arrangement of cells may become as shown in the 16-cell matrix on the right. Because of its property of self-similarity, the model of de Wijs was recognized to be a multifractal by Mandelbrot $(1983,1989)$ who adopted this approach for applications to the Earth's crust (also see Introduction).

Figure $2 \mathrm{~b}$ also shows Turcotte's variant of this approach: after each subdivision, only the half with larger concentration in further subdivided into halves with concentration values equal to $(1+d) \xi$ and $(1-d) \xi$. This simplifies the process as illustrated for 16 cells in 2-D space. At each stage of this process the concentration values have a Pareto-type frequency distribution. In analogy with Turcotte's (1997) derivation for blocks in 3-D space, it can be shown that a fractal dimension equal to $D=2 \times \log _{2}(1+d)$ can be defined for this process. The final element concentration map has only one maximum value.

Figure 3 shows a logbinomial pattern for $d=0.4$ and $k=14$. Increasing the number of subdivisions for the model of de Wijs (as in Fig. 2) to 14 resulted in the $128 \times 128$ pattern shown in which values greater than 4 were truncated. The frequency distribution of all $2^{14}$ values is logbinomial and approximately lognormal except in the highest-value and lowest value tails that are thinner than lognormal. When the number of subdivisions becomes large, the end product cannot be distinguished from that of multiplicative cascade models in which the dispersion index $D$ is modeled as a continuous random variable with mathematical expectation equal to 1 instead of the Bernoulli variable allowing the values $+d$ and $-d$ only (Agterberg, 2007). The lognormal model may provide good approximations for regional background distributions of trace elements.

Figure 4 is a mosaic of four patterns resulting from Turcotte "fractal" cascades with $d=0.4$ and $k=12$; vertical scale is logarithmic (base 10). Contrary to the multimodal logbinomial patterns, the Turcotte model develops a single peak only. However, the same cascade could have been operative in different parts of a study area. If the index of dispersion $(d)$ remains the same for all cascades, their combined frequency distribution after many subdivisions for each cascade would satisfy a single Pareto distribution plotting as a straight-line with slope determined by $d$.

\subsection{Self-similarity and the multifractal paradox}

De Wijs (1951) already derived an equation for the variance of logarithmically transformed element concentration values. 
It can be written in the form:

$\sigma^{2}=\frac{k}{4}(\ln \eta)^{2}$

where $\sigma^{2}$ is the logarithmic variance (base e) of the concentration values, and $\eta=(1+d) /(1-d)$. Equation (1) follows directly from the well-known equation for the variance of a binomial distribution with $p=1 / 2$ that is equal to $k / 4$, taking account of the fact that the spacing between ordered values along the logarithmic scale is equal to $\ln \eta$.

In principle, the value of $k$ could be made infinitely large. According to Eq. (1), the logarithmic variance then also becomes infinitely large and the frequency distribution of the element concentration values would cease to exist. Application of the method of moments in multifractal analysis results in a multifractal spectrum that is a limiting form for infinitely large $k$. The frequency distribution corresponding to this limiting form can not exist in reality because it has infinitely large variance. In practice, any set of element concentration values for very small blocks of rock collected from a very large environment has a frequency distribution with finite logarithmic variance.

Suppose that the generating process of subdividing blocks under the same dispersion index $(d)$ ceases to be operative for blocks that are larger than the very small blocks used for chemical analysis. This would imply that $d$ at the regional scale does not apply at the local scale. In practical applications (also see examples later in this section), $d$ locally becomes either zero or much smaller than $d$ at the regional scale. Under these conditions, an apparent maximum number of subdivisions $N(<k)$ can be estimated. Self-similarity at scales exceeding a critical lower limit then results in a model of de Wijs with three parameters: $\xi, d$, and $N$.

A multifractal method that can be used for estimating $d$ was proposed by Agterberg (2001a, b, 2007). It uses the method of moments as originally described by Evertsz and Mandelbrot (1992) and clearly explained in several textbooks including those by Feder (1988) and Falconer (2003). A brief summary is as follows. When the mass of a quantity is measured in cells, power moment sums can be formed by raising all mass measurements to powers $q$ and summing over all cells. A property of self-similar multifractals is that power moment sums are related to cell side according to power laws with mass exponents $\tau(q)$ (cf. Cheng and Agterberg, 1996). In two-dimensional applications, each cell mass measure is the product of cell concentration value and cell area. If the condition of self-similarity is satisfied, any mass exponent $\tau(q)$ can be estimated as the slope of the best-fitting straightline for a specific value of $q$. The first derivative of $\tau(q)$ with respect to $q$ provides an estimate of the so-called singularity $\alpha(q)$. The singularity $\alpha$ also can be mapped (Cheng, 1999; Cheng and Agterberg, 2007). Strictly speaking, a cascade does not result in point-wise convergence to any value of $\alpha$ because it performs a random walk at any scale. Instead of values at points, the local singularities are average values of $\alpha$ within small cells.

\subsection{Estimation of dispersion index (d) for lognormal} "background"

The singularity has its minimum value $\left(\alpha_{\min }\right)$ for the cell with the largest possible concentration value. Validity of the model of de Wijs for larger concentration values results in:

$$
\lim _{q \rightarrow \infty} \frac{d \tau(q)}{d q}=\alpha_{\min }=\log _{2}\left(\frac{\eta+1}{\eta}\right)^{2}
$$

In 2-dimensional applications, chemical element measures can be created by multiplying average cell concentration values by cell area. Raising these measures to relatively high powers $q$ filters out the influence of smaller concentration values. Thus our estimate of $d$ is primarily based on parameters estimated from the relatively large concentration values of an element. The lower-value tails of the observed frequency distributions could be mixtures of separate populations, and the exact nature of these mixtures can be left undetermined.

If the model of de Wijs is satisfied, the mass exponents $\tau(q)$ become linearly related to $q$ when $q$ is large and the parameter $d$ can be estimated from the slope of the resulting straightline. Together with the estimate of logarithmic variance this yields an estimate of apparent maximum number of subdivisions $N$ when Eq. (1) is used. It is noted that $d$ also can be estimated from the multifractal spectrum which normally is the end product of applications of the method of moments. However, Eq. (2) may produce better results more quickly (Agterberg, 2007).

In an application of this approach to glacial till samples from a $625 \mathrm{~km}$ by $625 \mathrm{~km}$ area in southern Saskatchewan, the lognormal provided good approximations for gold with $\sigma^{2}(\mathrm{Au})=5.61$, and arsenic with $\sigma^{2}(\mathrm{Ar})=0.131$, respectively (cf. Agterberg, 2007). Coefficients of dispersion estimated by Eq. (2) were $d(\mathrm{Au})=0.433$ and $d(\mathrm{Ar})=0.069$. Although the frequency distributions for gold and arsenic in this application were greatly different, estimates of apparent numbers of subdivisions $(N)$ using Eq. (1) were approximately equal (26.1 and 27.5, respectively). The smallest cell in these applications would measure approximately $50 \mathrm{~m}$ on a side, significantly exceeding horizontal area of original till samples. In this example, the model of de Wijs is valid on a regional scale with approximately constant coefficient of dispersion (d) with element concentration maps similar to Fig. 3. However, in other applications, the largest element concentration values do not fit in with a lognormal model.

\subsection{Accelerated dispersion model}

A relatively simple modification of the model of de Wijs that results in thicker than lognormal frequency distribution tail is to assume that the coefficient of dispersion $d$ increases as 


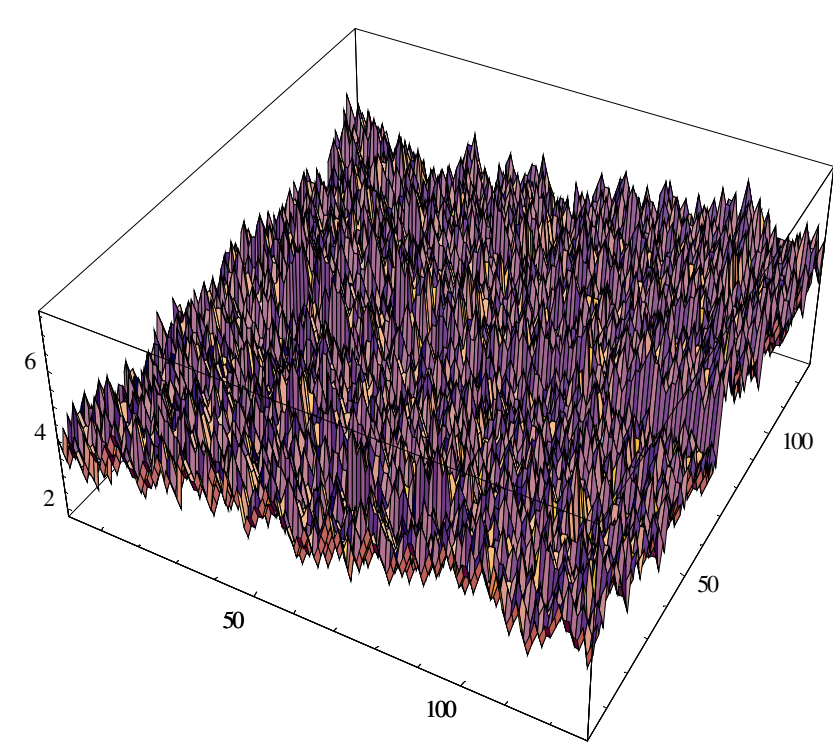

Fig. 5. Realization of accelerated dispersion model for $d_{0}=0.4$, $N=14$, and $p=0.01$. Vertical scale is for $4+\log _{10}$ (Value).

a function of element concentration value $\xi$. For example, it could be assumed that the first derivative of a chemical element's dispersion coefficient is a linear function of $\xi$ so that $d=d_{0} \exp (p \times \xi)$ where $p$ is a constant. Setting $p=0.01$ and re-running the experiment previously resulting in Fig. 3 $\left(d_{0}=0.4 ; N=14\right)$ yielded the pattern for $(\xi+4)$ shown in Fig. 5 where the vertical scale is logarithmic. The logarithmically transformed concentration values of Fig. 5 are normally distributed except for the largest values. Figure 6 is a comparison of the largest log-concentration values with those arising when there would be no acceleration of dispersion. Values with $\log _{10}(\xi) \approx 2$ are larger than expected in comparison with patterns such as Fig. 3 resulting from the model of de Wijs with $p=0$. Only relatively few very large values emerge from the approximately lognormal background if $p$ is small.

The accelerated dispersion model provides a possible explanation for occurrence of anomalously high concentration values. Applicability of this model in practice remains to be investigated.

\subsection{Pareto distributions}

Turcotte's cascade model is a modification of the preceding multifractal-generating cascade. Only cells with largest concentration value during a previous subdivision are further subdivided into parts with different element concentration values. The random pattern then has a single maximum only. Thus it is assumed that the same type of cascade was operational at $n$ different random locations generating a pattern with $n$ maxima. The frequency distribution of the concentration values then remains the same except for enlargement of all frequencies by the factor $n$. Element concentrations

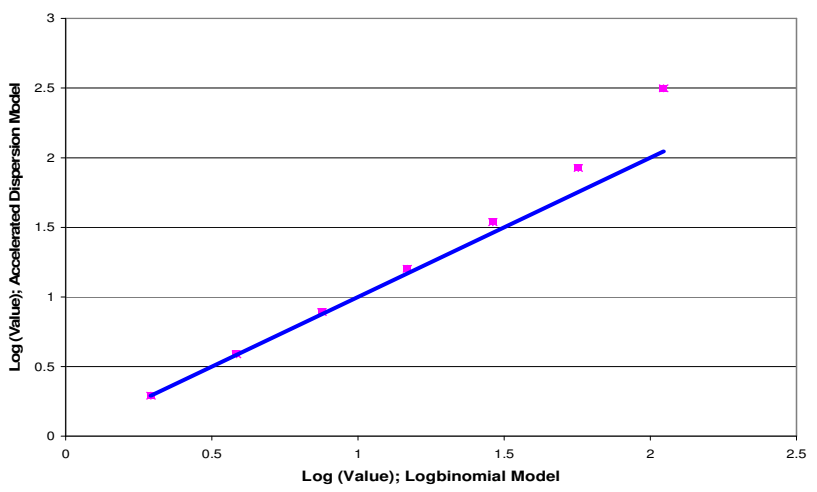

Fig. 6. Comparison of largest logarithmically transformed concentration values (base 10) in experiment of Fig. 5.

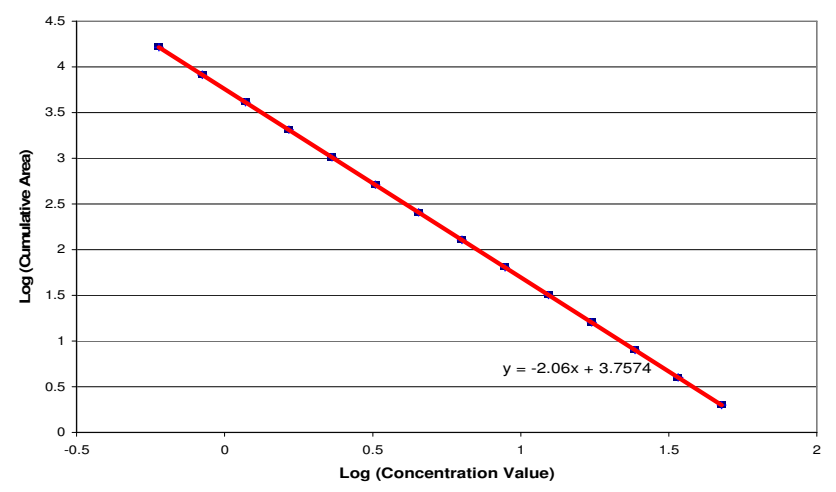

Fig. 7. C-A (Concentration-Area) plot for Turcotte's fractal model with $d=0.4$ and $N=14$ (Log base 10$)$.

generated by Turcotte's cascade satisfy a Pareto distribution which is associated with a fractal instead of a multifractal. The slope $\beta$ of the straight-line representing this Pareto distribution on $\log -\log$ paper satisfies $\beta=-1 / \log _{2}(1+d)$. Figure 7 is a C-A diagram for the Turcotte model with $d=0.4$ and $N=14$. Consequently, $\beta=-2.060$.

The following computer simulation experiments illustrate that an unbiased estimate of the Pareto parameter can be obtained in the hypothetical situation of a study area where background satisfies the model of de Wijs with overall average concentration value equal to 0.1 and $d=0.3$. Suppose that one or more Turcotte cascades with overall average value equal to 1.0 and $d=0.4$ (cf. Fig. 4) are superimposed on this background. Figure 8 is a C-A diagram for this hypothetical situation. The pattern in this diagram is approximately linear with slope of approximately -2 . It was obtained by random sampling of concentration values resulting from the de Wijs and Turcotte cascades and adding these values together.

Suppose now that the Turcotte cascades were operational in only $25 \%$ of the area. Use of Cheng's (2003) method of piecemeal fitting of successive straightline segments in a C-A diagram indicates that the largest concentration values 


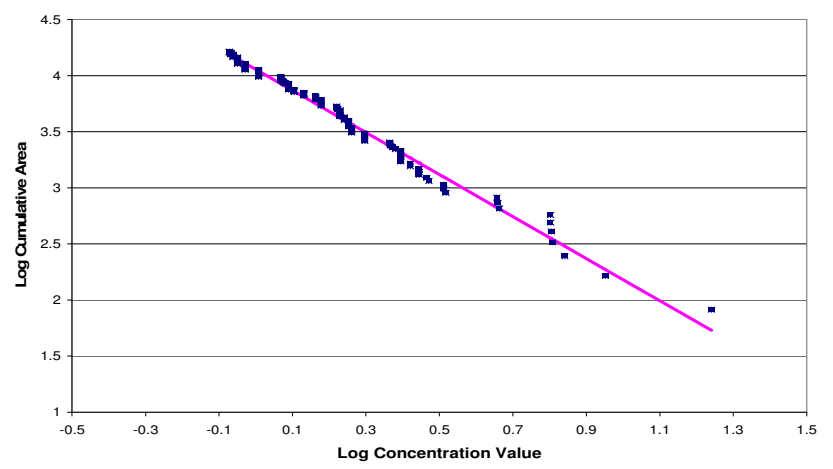

Fig. 8. C-A plot for logbinomial background with mean $m=0.1$ and $d=0.3$ with superimposed Turcotte anomalies with $m=1.0$ and $d=0.4$ (Log base 10).

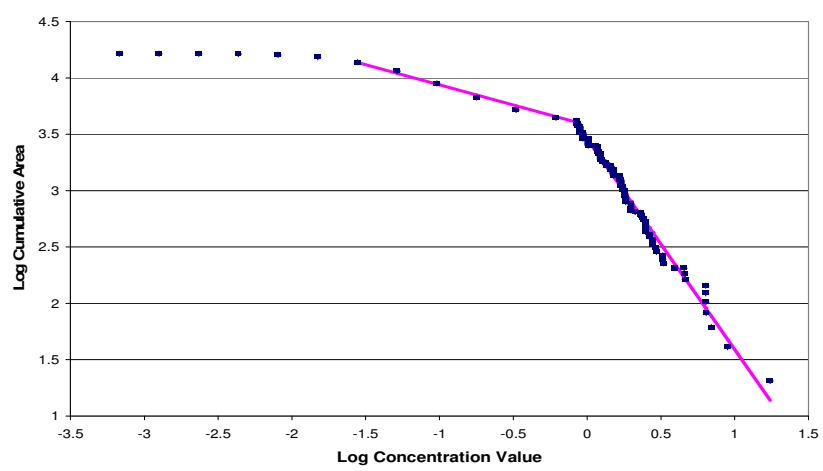

Fig. 9. C-A plot for lognormal background with superimposed Turcotte anomalies as in Fig. 8 but Turcotte anomalies restricted to $25 \%$ of total area (Log base 10).

approximately fall on a straightline with slope equal to -2 as in Figs. 7 and 8. Consequently, the estimated value of $d$ is 0.4. These experiments (Figs. 8-9) illustrate that unbiased estimates of this type can be obtained irrespective of how many Turcotte cascades there were in the area or how much of the study area consists of approximately lognormal background without anomalies. Figure 10 is plot of the frequency density values for the second experiment. The smaller peak on the right corresponds to the line-segment for largest concentration values in Fig. 9.

2.6 Practical example of dispersion index $(d)$ estimation for Turcotte's model

Suppose a measure $\mu$ of the amount of a chemical element in a square cell measuring $\varepsilon \mathrm{km}$ on a side satisfies $\mu=c \times \varepsilon^{\alpha}$ where $c$ is a constant, and $\alpha$ is the singularity also known as Hölder exponent (Mandelbrot, 1989; Evertsz and Mandelbrot, 1992); then $\alpha$ can be estimated by measuring the slope of the straight line in a $\log -\log$ plot of $\mu$ against $\varepsilon$. In this 2-dimensional application of multifractal theory, $\mu=\xi \times \varepsilon^{2}$ where $\xi=c \times \varepsilon^{\alpha-2}$ represents average element concentration

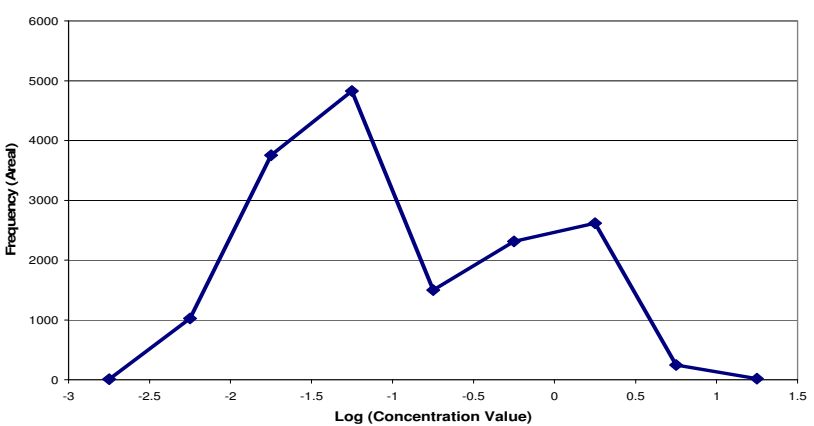

Fig. 10. Frequencies of lognormal-Pareto mixture plotted as C-A diagram in Fig. 9 (Log base 10).

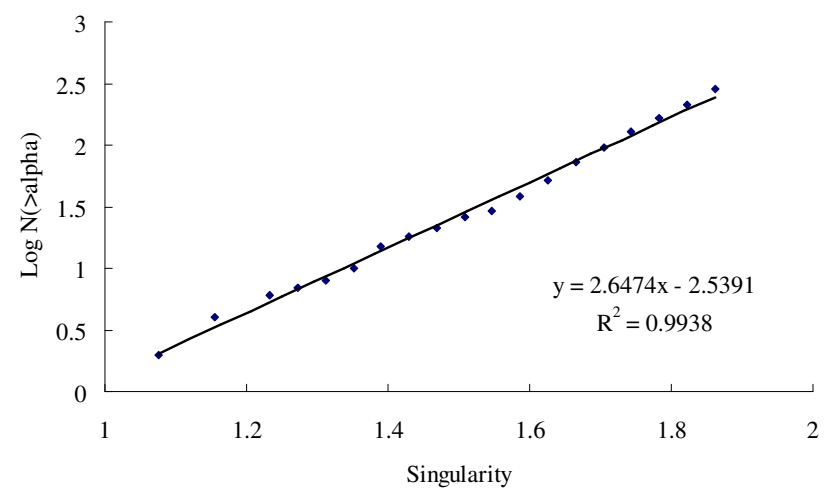

Fig. 11. Natural logs of areal frequencies for local singularities of arsenic in Gejiu area, Yunnan Province, China (after Cheng, 2007; Cheng and Agterberg, 2007).

value in the cell. If element concentration values for samples taken at the surface of a study area are realizations of a stationary random variable with constant population mean, then $\alpha=2$ representing non-singularity. "Singular" locations (where $\alpha<2$ ) may indicate anomalous enrichment of the chemical element.

Examples of local singularity mapping are given in Cheng (2007) and Cheng and Agterberg (2007) for various elements in stream sediments from the Gejiu area, Yunnan Province, China. Like several other chemical elements, arsenic in these surficial deposits shows two types of anomalies. The arsenic local singularity map for arsenic shows many relatively small anomalies (where $\alpha<2$ ) across the entire Gejiu area. A significant fraction of these anomalies is spatially correlated with occurrences of (mined and unmined) mineral deposits. The arsenic concentration values are highest in the eastern part of the area. Together the highest values describe a large irregularly shaped anomaly that is probably caused by mining activities restricted to this part of the area.

Figures 11 and 12 show frequencies of As local singularities and As concentration values, respectively. Suppose that parameters describing the two preceding anomaly types are 


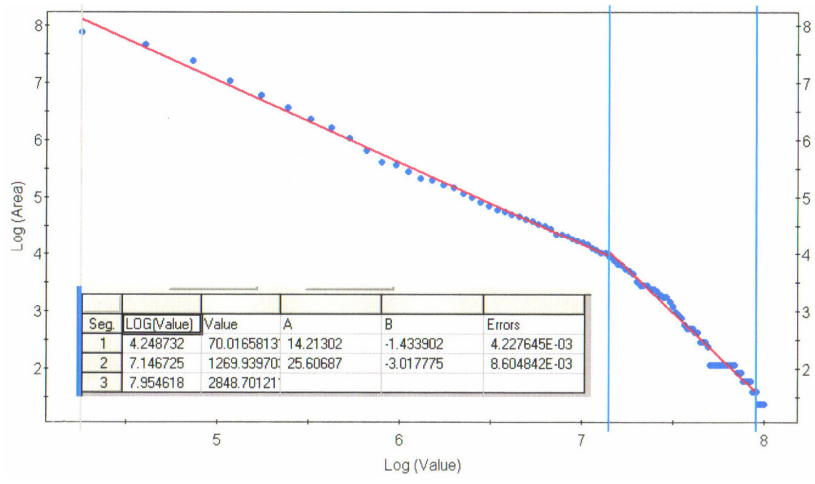

Fig. 12. C-A plot for As in Gejiu area (from Cheng and Agterberg, 2007). Point pattern was automatically subdivided into straightline segments with statistics in box; A = intercept; $\mathrm{B}=$ slope; errors represent variances of slope estimates; Log base 10; cf. Cheng, 2003).

identified by the subscripts 1 (for local singularity anomalies) and 2 (for the high-concentration anomaly). From $\beta_{2}=-3.0178$ (estimated slope of best-fitting line in Fig. 12), it follows immediately that $d_{2}$ (As) $=0.258$. From $\xi=c \times \varepsilon^{\alpha-2}$ with $\varepsilon=2 \mathrm{~km}$ in this application, it follows that estimated slope of straight-line in Fig. $11(=-2.6474)$ provides an estimate of $\beta_{1}=-8.7945$. Consequently, $d_{1}$ (As) $=0.082$.

Suppose that $\beta$ is a parameter estimated by the slope of the best-fitting straight-line on a C-A plot. Then this estimate can be converted into either the fractal dimension $D$ $(=-2 / \beta)$ or into the index of dispersion $d\left(=2^{-D / 2}-1\right)$ characterizing the non-linear process. In terms of Fig. 2: if a block with high-concentration value $(\xi)$ is divided into two halves, the concentration values of the halves are, on average, equal to $(1+d) \times \xi$ and $(1-d) \times \xi$, respectively. Thus a higher index of dispersion means stronger spatial variability. The small anomalies (where $\alpha<2$ ) with $d_{1}(\mathrm{As})=0.082$ have lower dispersion index than the broad regional anomaly restricted to the eastern part of the area with $d_{2}(\mathrm{As})=0.258$. Several other elements (tin, copper, silver, gold, cadmium, cobalt, iron, nickel lead, and zinc) show anomalies similar those for arsenic (Cheng and Agterberg, 2007). The first type (local singularities) is useful for exploration because it provides indicators for buried ore-bodies. The second type helps to describe regional pollution due to mining activities. The shapes of two kinds of anomalies (1 and 2) are markedly different, and this probably is the main reason that a clear distinction could be made between the two underlying enrichment processes (proximity to buried mineral deposits and pollution due to mining activities) in this example of application.

\section{Concluding remarks}

Multifractal modeling is well established in geophysics particularly with respect to processes taking place in the atmosphere including cloud formation and rainfall. Cascade dy- namics play an important role in nonlinear process modeling. Many short-term solid-Earth processes including earthquakes, landslides and flooding exhibit non-linear characteristics. Lognormality and power-law behavior also are associated with mineral deposits (metal and hydrocarbon accumulations) and secondary processes such as inclusion of toxic and nontoxic elements in stream sediments.

Because of the great variety and irregular exposure of rock formations it is difficult to apply non-linear process modeling to solid-Earth. However, multifractal modeling adds new tools to conventional approaches in geochemistry and economic geology. This paper was primarily concerned with mixtures of non-linear processes. It was argued that regional background concentration values of elements may approximately satisfy a three-parameter model of de Wijs resulting in lognormality. Anomalous accumulations of metals may have been superimposed on background later according to non-linear processes resulting in power-law distributions of element concentration values.

Acknowledgements. Thanks are due to Q. Cheng (York University, Toronto) and S. Lovejoy (McGill University, Montreal) for helpful suggestions.

Edited by: Q. Cheng

Reviewed by: one referee

\section{References}

Agterberg, F. P.: Multifractal modeling of the sizes and grades of giant and supergiant deposits, Int. Geol. Rev., 37, 1-8, 1995.

Agterberg, F. P.: Multifractal simulation of geochemical map patterns, in: Geologic modeling and simulation: Sedimentary Systems, edited by: Merriam, D. F. and Davis, J. C., Kluwer, New York, 327-346, 2001a.

Agterberg, F. P.: Aspects of multifractal modeling: Proceedings of IAMG Annual Meeting, Cancun, September, CD-Rom, 2001b.

Agterberg, F. P.: New applications of the model of de Wijs in regional geochemistry, Math. Geol., Preprint, 2007.

Ahrens, L. H.: A fundamental law of geochemistry, Nature, 712, 1148, 1953.

Aitchison, J. and Brown, J. A. C.: The lognormal distribution, Cambridge Univ. Press, 1957.

Allègre, C. J. U. and Lewin, E.: Scaling laws and geochemical distributions, Earth Planet. Sci. Lett., 132, 1-13, 1995.

Brinck, J. W.: The geochemical distribution of uranium as a primary criterion for the formation of ore deposits, in: Chemical and physical mechanisms in the formation of uranium mineralization, geochronology, isotope geology and mineralization, International Atomic Energy Agency, Proc. Ser. STI/PUB/374, $21-$ 32, 1974

Cheng, Q.: Multifractal modeling and spatial analysis with GIS: gold potential estimation in the Mitchell-Sulphurets Area, northwestern British Columbia, University of Ottawa, Canada, doctoral thesis, 1994.

Cheng, Q.: Multifractality and spatial statistics, Computers \& Geosci., 25, 949-961, 1999. 
Cheng, Q.: GeoData Analysis System (GeoDAS) for Mineral Exploration and Environmental Assessment, User's Guide, York University, Toronto, Canada, 2003.

Cheng, Q.: Multifractal distribution of eigenvalues and eigenvectors from 2D multiplicative cascade multifractal fields, Math. Geol., 37, 915-927, 2005.

Cheng, Q.: Mapping singularities with stream sediment geochemical data for prediction of undiscovered mineral deposits in Gejiu, Yunnan Province, China, Ore Geol. Rev., in press, 2007.

Cheng, Q. and Agterberg, F.P.: Multifractal modeling and spatial statistics, Math. Geol., 28(1), 1-16, 1996.

Cheng, Q. and Agterberg, F. P.: Singularity analysis of ore-mineral and toxic trace elements in stream sediments, Computers \& Geosci., accepted, 2007.

Cheng, Q., Agterberg, F. P., and Ballantyne, S. B.: The separation of geochemical anomalies from background by fractal methods, J. Geochem. Explor., 51(2), 109-130, 1994.

De Wijs, H. -J.: Statistics of ore distribution, part I, Geologie en Mijnbouw, 13, 365-375, 1951.

Evertsz, C. J. G. and Mandelbrot, B. B.: Multifractal measures, Appendix B, in: Chaos and Fractals, edited by: Peitgen, H.-O., Jurgens, H., and Saupe, D., Springer-Verlag, New York, 922-953, 1992.

Falconer, K.: Fractal geometry - mathematical foundations and applications (2nd ed.), Wiley, New York, 2003.

Feder, F.: Fractals, Plenum, New York, 1988.

Garrett, R. G.: Geochemical abundance models: an update, 1975 to 1987, U.S. Geological Survey Circular 980, 207-220, 1986.

Harris, D. P.: Mineral resources appraisal, Clarendon Press, Oxford, 1984.

Lovejoy, S. and Schertzer, D.: Multifractal analysis techniques and the rain and cloud fields from $10^{-3}$ to $10^{6} \mathrm{~m}$, in: Non-linear variability in geophysics, edited by: Schertzer, D. and Lovejoy, S., Kluwer, Dordrecht, 111-144, 1991.
Mandelbrot, B. B.: The fractal geometry of nature (updated and augmented edition), W.H. Freeman and Company, New York, 1983.

Mandelbrot, B. B.: Multifractal measures, especially for the geophysicist, Pure Appl. Geophys., 131, 5-42, 1989.

Over, T. M. and Gupta, V. K.: A space-time theory of mesoscale rainfall using random cascades, J. Geophys. Res., 101, 2631926331, 1996.

Schertzer, D. and Lovejoy, S.: The dimension and intermittency of atmospheric dynamics Multifractal cascade dynamics and turbulent intermittency, in: Turbulent Shear Flow 4, edited by: Launder, B., Springer-Verlag, New York, pp. 7-33, 1985.

Schertzer, D. and Lovejoy, S.: Physical modeling and analysis of rain and clouds by anisotropic scaling of multiplicative processes, J. Geophys. Res., 92, 9693-9714, 1987.

Schertzer, D., Lovejoy, S., Schmitt, F., Chigirinskaya, Y., and Marsan, D.: Multifractal cascade dynamics and turbulent intermittency, Fractals, 5, 427-471, 1997.

Sinclair, A. J.: A fundamental approach to threshold estimation in exploration geochemistry: probability plots revisited, J. Geochem. Explor., 41(1), 1-22, 1991.

Turcotte, D. L.: Fractals and chaos in geology and geophysics (2nd ed.), Cambridge Univ. Press, 1997.

Turcotte, D. L.: Fractals in petrology, Lithos, 65(3-4), 261-271, 2002.

Veneziano, D. and Furcolo, P.: Marginal distribution of stationary multifractal measures and their Haar wavelet coefficients, Fractals, 11(3), 253-270, 2003.

Veneziano, D. and Langousis, A.: The maximum of multifractal cascades: exact distribution and approximations, Fractals, 13(4), 311-324, 2005.

Vistelius, A. B.: The skew frequency distributions and the fundamental law of geochemistry, J. Geol., 69, 703-728, 1960. 